\title{
Role of cognitive reserve in cognitive variability in euthymic individuals with bipolar disorder: cross-sectional cluster analysis
}

Dimosthenis Tsapekos, Rebecca Strawbridge, Tim Mantingh, Matteo Cella, Til Wykes and Allan H. Young

\section{Background}

People with bipolar disorder have moderate cognitive difficulties that tend to be more pronounced during mood episodes but persist after clinical remission and affect recovery. Recent evidence suggests heterogeneity in these difficulties, but the factors underlying cognitive heterogeneity are unclear.

\section{Aims}

To examine whether distinct cognitive profiles can be identified in a sample of euthymic individuals with bipolar disorder and examine potential differences between subgroups.

\section{Method}

Cognitive performance was assessed across four domains (i.e. processing speed, verbal learning/memory, working memory, executive functioning) in 80 participants. We conducted a hierarchical cluster analysis and a discriminant function analysis to identify cognitive profiles and considered differences in cognitive reserve, estimated cognitive decline from premorbid cognitive functioning, and clinical characteristics among subgroups.

\section{Results}

Four discrete cognitive profiles were identified: cognitively intact $(n=25 ; 31.3 \%)$; selective deficits in verbal learning and memory $(n=15 ; 18.8 \%)$; intermediate deficits across all cognitive domains $(n=30 ; 37.5 \%)$; and severe deficits across all domains $(n=10$;
12.5\%). Cognitive decline after illness onset was greater for the intermediate and severe subgroups. Cognitive reserve scores were increasingly lower for subgroups with greater impairments. A smaller proportion of cognitively intact participants were using antipsychotic medications compared with all other subgroups.

\section{Conclusions}

Our findings suggest that individuals with cognitively impaired profiles demonstrate more cognitive decline after illness onset. Cognitive reserve may be one of the factors underlying cognitive variability across people with bipolar disorder. Patients in the intermediate and severe subgroups may be in greater need of interventions targeting cognitive difficulties.

\section{Keywords}

Bipolar disorder; clustering; cognitive profiles; cognitive reserve: cognitive remediation.

\section{Copyright and usage}

(C) The Author(s), 2020. Published by Cambridge University Press on behalf of the Royal College of Psychiatrists. This is an Open Access article, distributed under the terms of the Creative Commons Attribution licence (http://creativecommons.org/ licenses/by/4.0/), which permits unrestricted re-use, distribution, and reproduction in any medium, provided the original work is properly cited.
Bipolar disorder is a mental health condition characterised by recurrent episodes of depression and (hypo)mania. Recent findings suggest that people with bipolar disorder experience moderate cognitive impairments, $0.5-1$ standard deviation (s.d.) below the normative mean, which are more pronounced during mood episodes but persist after the symptoms remit, during periods of euthymia. ${ }^{1,2}$ Deficits appear across multiple domains, including processing speed, verbal memory and executive functioning. ${ }^{3}$ Considerable evidence suggests that these impairments affect daily life functioning, possibly independent of mood symptoms. ${ }^{4}$ Findings indicate that cognitive difficulties are present in a proportion of patients with bipolar disorder, ${ }^{5,6}$ and more recently this heterogeneity has been characterised through the identification of different profiles of cognitive difficulties.

The evidence suggests that there are three or four discrete and coherent profiles, one cognitively intact and comparable to the general population, plus one or two subgroups presenting with selective moderate impairments, and a globally impaired subgroup with severe impairments across cognitive domains. ${ }^{7}$ Similar findings have been reported from studies with cross-diagnostic samples involving people with different diagnoses across the psychosis spectrum. ${ }^{8,9}$

There is also a limited understanding of the factors underlying $\operatorname{cog}$ nitive heterogeneity in bipolar disorder. An aspect of cognitive heterogeneity remaining unexplored is whether cognitive clusters represent different degrees of cognitive decline following illness onset and the extent of this putative decline across subgroups. A recent study with a cross-diagnostic sample defined cognitive decline as the discrepancy between current cognitive performance and premorbid IQ and reported evidence for a cluster characterised by a large cognitive decline, but limited changes for the other subgroups. ${ }^{10}$

Cognitive reserve might be a factor underlying this difference in cognitive decline between clusters. ${ }^{11}$ It reflects resilience to brain pathology by minimising its effect on behavioural outcomes, such as symptom manifestation or cognitive dysfunction. ${ }^{12,13}$ Several proxy measures of cognitive reserve have been suggested, including years of education, occupational attainment and measures of vocabulary knowledge or reading abilities. ${ }^{14}$ The concept of reserve has been extensively explored in neurological and psychiatric disorders such as dementia, multiple sclerosis and schizophrenia, representing individual differences in the capacity to compensate for age- and illness-related cognitive decline in the presence of neuropathology. ${ }^{14-16}$ Previous research in bipolar disorder suggests an association of cognitive reserve with cognitive performance, ${ }^{17}$ but this has not been explored in the context of cognitive heterogeneity, particularly whether and to what extent cognitive differences between putative subgroups can be explained as a function of cognitive reserve.

Illness-history variables, such as the type and the number of previous mood episodes, have been investigated as putative underlying factors of cognitive heterogeneity. However, previous studies have reported inconsistent findings on the association of clinical characteristics and medication use with the profile of cognitive impairment. ${ }^{7}$ The inclusion of participants partially remitted or with subsyndromal 
symptoms may have resulted in these mixed findings between studies. Hence, it remains unclear whether cognitive performance across cognitive clusters is affected by patient differences in illness-related characteristics.

Here, we examine whether discrete cognitive profiles can be identified within a cohort of euthymic patients with bipolar disorder and test the hypothesis that cognitive reserve will differ between subgroups. We anticipate replicating the findings of previous cluster-analytic studies reporting on euthymic participants ${ }^{18,19}$ and we use independent cognitive measures to internally validate the identified clusters. Cognitive reserve and estimated postmorbid cognitive decline are examined as factors underlying potential differences in the cognitive course of putative subgroups. Differences in clinical characteristics are also examined among clusters. Parsing cognitive heterogeneity in bipolar disorder is important for delivering targeted interventions and potentially improving outcomes. A better understanding of the underlying factors associated with cognitive clustering may also inform intervention strategies, as well as help clinical services recognise earlier or more efficiently which patients require cognitive treatment.

\section{Method}

\section{Study design}

This is a cross-sectional secondary analysis of baseline data from the Cognitive Remediation in Bipolar (CRiB) study, a feasibility trial comparing cognitive remediation with treatment as usual in people with bipolar disorder. ${ }^{20,21}$ Written informed consent was obtained from all participants prior to inclusion. Baseline assessments were undertaken before random allocation to the treatment or the control group. The authors assert that all procedures contributing to this work comply with the ethical standards of the relevant national and institutional committees on human experimentation and with the Helsinki Declaration of 1975, as revised in 2008. All procedures involving human patients were approved by the City Road \& Hampstead NHS Research Ethics Committee (reference 15/LO/1557).

\section{Participants}

The sample comprised 80 out-patients with a DSM-5 diagnosis of bipolar disorder. All participants were fluent in English and aged between 18 and 65 years. Bipolar disorder subtype and eligibility were confirmed using the Mini International Neuropsychiatric Interview. ${ }^{22}$ Participants were free of acute mood symptoms for at least 1 month prior to inclusion. Remission at screening stage was defined using cut-off scores of $\leq 7$ on the 17-item Hamilton Rating Scale for Depression (HRSD) $)^{23}$ and the Young Mania Rating Scale (YMRS). ${ }^{24}$ Participants with a diagnosed neurological disorder or personality disorder and those misusing or dependent on alcohol or illicit substances over the previous 6 months were excluded.

\section{Measures}

\section{Clinical assessment}

A structured interview was used to collect information on demographic characteristics, illness-history variables and current medication use. Mood symptoms were assessed using the HRSD for depressive and the YMRS for hypomanic/manic symptoms.

\section{Cognitive assessment}

Clustering measures. Participants were administered an extensive cognitive battery in a standardised order. The battery included eight tests to be used as clustering tests: the Hotel test, ${ }^{25}$ Wechsler
Memory Scale Fourth Edition (WMS-IV) Verbal Paired Associates immediate and delayed recall (VPA I and II), ${ }^{26}$ Wechsler Abbreviated Scale of Intelligence Second Edition (WASI-II) Matrix Reasoning subset, ${ }^{27}$ Wechsler Adult Intelligence Scale Fourth Edition (WAIS-IV) Digit Span, Digit Symbol-Coding and Symbol Search, ${ }^{28}$ and the F-A-S letter verbal fluency test from the DelisKaplan Executive Function System. ${ }^{29}$

Non-clustering measures. Two measures of general cognitive performance were used for the internal validation of the emerging cognitive subgroups. ${ }^{10}$ Participants were administered the Montreal Cognitive Assessment (MoCA), ${ }^{30}$ a compact screening instrument assessing multiple cognitive domains and giving a single score. We also used the cognitive subscale of the Functional Assessment Short Test (FAST), ${ }^{31}$ a clinician-rated measure examining cognitive functioning in the context of daily life situations and activities, with higher scores indicating greater difficulties.

IQ measures. Two intelligence measures were administered: an estimate of premorbid IQ, the Test of Premorbid Function (TOPF), ${ }^{32}$ and an estimate of verbal IQ, the Vocabulary subtest of the WASI-II. ${ }^{27}$ Both represent cognitive reserve as measures of crystallised intelligence and have been shown to be resistant to the effects of ageing or illness-related decline. ${ }^{33}$

\section{Statistical analysis}

Despite a lack of consensus on the required sample size to achieve adequate statistical power in cluster analysis, a minimum sample size of $2^{k}$ (where $k$ is the number of clustering variables) has been suggested, although the ideal sample size would be five times this number. ${ }^{34}$ On the basis of this recommendation and our sample of 80 participants, we considered four clustering variables in this study.

All statistical analyses were conducted using the Statistical Package for Social Sciences (SPSS), version 25 for Windows, with a statistical significance of $P<0.05$ (two-tailed) for all tests. The distributions were checked for normality across all measures using the Shapiro-Wilk test and log transformation was applied to conform non-normally distributed variables. Descriptive statistics were computed for all variables.

\section{Cognitive domains for clustering}

Raw scores of cognitive tests were transformed to demographically corrected standardised scores $(z$-scores; mean 0 , s.d. $=1)$ based on the normative data for each test as provided by manuals. The Hotel test $z$-scores were inverted to be consistent with the direction of other measures, since for this test higher scores represent poorer performance. We computed $z$-scores for four cognitive domains: processing speed using the average scores of the Digit SymbolCoding and the Symbol Search; working memory with the Digit Span forward, backward and sequencing scores; verbal learning and memory using the average scores of the VPA I and VPA II; and executive functioning using the average scores of the Hotel test, Matrix Reasoning and F-A-S letter verbal fluency test. A composite score of current global cognition was computed for each participant by averaging the $z$-scores of all the tests used for the clustering domains.

\section{Identifying and validating cognitive clusters}

The four cognitive domains were entered into a hierarchical cluster analysis to identify subgroups with homogeneous cognitive profiles according to their performance in these domains. Following Burdick et al, ${ }^{11}$ we used Ward's linkage as the clustering method and squared Euclidean distance to estimate similarities between cases. To validate the initial clustering and evaluate the accuracy 
of participant allocation across clusters, we conducted a discriminant function analysis (DFA). The optimal number of clusters was determined on the basis of visual inspection of the dendrogram and the DFA coefficients. DFA examines the predictive power of different domain scores for every subgroup identified in the hierarchical cluster analysis and determines the probability of classification into a certain subgroup for every participant on the basis of these scores.

To estimate in which domains and to what extent these subgroups are distinct, cognitive profiles were compared to each other using multivariate analysis of covariance (MANCOVA) with least significant difference (LSD) for pairwise comparisons. Age was used as a covariate since age differences were evident between subgroups. For MANCOVAs with a significant main effect, post hoc comparisons between subgroups were corrected for multiple testing using a false discovery rate (FDR) of 5\%.

Given that the cluster analysis was applied to differentiate participants on the basis of their cognitive performance, we anticipated significant between-group differences that would internally validate the cognitive clustering of the sample. Cohen's $d$ effect sizes (defined as the mean group difference divided by the pooled s.d. and corrected for unequal group sizes) were calculated for significant post hoc comparisons. To further validate within the sample that the emerging cognitive profiles are truly distinct and not just artefacts of the measures used to identify them, subgroups were compared on non-clustering cognitive measures (MoCA and FAST cognitive subscale) using MANCOVA with age as a covariate and correcting for multiple comparisons with a $5 \% \mathrm{FDR}$.

\section{Estimating cognitive decline and cognitive reserve}

A proxy measure of postmorbid cognitive decline was estimated for each participant as the discrepancy between the current global cognitive performance (composite score) and the estimated premorbid IQ (TOPF score), following the methodology of previous studies. ${ }^{8,10}$ Both the global cognition composite and the TOPF score were standardised to the normative performance of healthy controls according to the validation data.

Cognitive reserve was estimated for each participant through a composite score of three proxy measures: years of education and the two intelligence indices, the TOPF and the Vocabulary subtest of the WASI-II, according to previous recommendations. ${ }^{15,16}$ Using a factor analysis, a single score was derived from these three variables for each participant. ${ }^{15}$ This factor score accounted for $59.8 \%$ of the shared variance in the three variables and was used as a measure of cognitive reserve in all subsequent analyses. To ensure that cognitive reserve is an estimate independent of ageing and illness progression, this score was correlated to participants' age, age at illness onset, illness duration and number of mood episodes.

Differences between subgroups in cognitive decline and cognitive reserve were examined using a MANCOVA (age as covariate) with an FDR of 5\% to correct for multiple comparisons.

\section{Comparisons on demographic and clinical characteristics}

Subgroup comparisons were conducted to examine differences in non-cognitive variables using chi-squared $\left(\chi^{2}\right)$ tests or analyses of variance (ANOVAs) with Games-Howell post hoc correction for unequal variances and small sample sizes. Identified clusters were compared on demographic characteristics, clinical history, current mood symptoms, use of medication and previous service use. To estimate the magnitude of potential differences, Cohen's $d$ was calculated using the same procedure. Cohen's $w$ effect sizes were calculated for categorical variables.

\section{Results}

\section{Sample characteristics}

Table 1 provides details on the demographic and clinical characteristics of sample. There were no missing data for any clinical variables or cognitive tests. Overall, the sample performed mildly below the normative scores in two domains: verbal learning and memory $(z=-0.26$, s.d. $=1.1)$ and executive functioning $(z=-0.22$, s.d. $=0.66)$. Even smaller differences were detected in processing speed $(z=-0.14$, s.d. $=0.72)$, working memory $(z=-0.08$, s.d. $=0.61)$ and global cognition $(z=-0.17$, s.d. $=0.61)$.

\section{Identifying cognitive clusters}

Inspection of the dendrogram and the agglomeration coefficients suggested a four-cluster solution as the most appropriate fit for the sample: a subgroup of 25 participants (31\%) remained cognitively intact, another subgroup of 15 participants (19\%) had a selective deficit in verbal learning and memory, a third subgroup of 30 participants (37.5\%) had intermediate impairment across all domains and a fourth subgroup of 10 participants (12.5\%) had severe global impairments. The intact cluster performed slightly above the normative mean in three domains (by $0.2-0.3$ s.d.) and 1 s.d. above the normative mean in verbal learning and memory. The second cluster showed a comparable performance to the intact subgroup in processing speed, working memory and executive functioning, but presented a selective impairment of 1 s.d. below the normative mean in verbal learning and memory. The intermediate cluster consistently performed $0.3-0.5$ s.d. below the

Table 1 Sample demographic and clinical characteristics $(N=80)$

\begin{tabular}{|c|c|c|}
\hline & Mean (s.d.) & $\begin{array}{l}\text { Minimum- } \\
\text { Maximum }\end{array}$ \\
\hline Age & $42.2(12.8)$ & 19-65 \\
\hline Education, years & $15.9(2.1)$ & $11-21$ \\
\hline Estimated premorbid IQ (TOPF) & $109.2(6.9)$ & $93-121$ \\
\hline Age bipolar disorder diagnosed, years & $30.9(11.7)$ & $16-61$ \\
\hline Diagnosis duration, years & $10.8(8.9)$ & $0-34$ \\
\hline Symptom duration, years & $21.5(12.7)$ & $1-56$ \\
\hline Duration of untreated illness, years & $10.7(9.6)$ & $0-44$ \\
\hline Number of depressive episodes & $12.4(14.3)$ & $1-91$ \\
\hline Number of (hypo)manic & $8.5(7.2)$ & $1-32$ \\
\hline Number of hospital admissions & $2.4(2.9)$ & $0-14$ \\
\hline Current euthymia, months & $13.8(24.6)$ & $1-168$ \\
\hline Number of current medications & $2.4(1.5)$ & $0-7$ \\
\hline $\begin{array}{l}\text { Number of psychological therapies } \\
\text { undertaken }\end{array}$ & $1.96(1.54)$ & $0-8$ \\
\hline HRSD-17 score & $3.3(2.2)$ & $0-7$ \\
\hline \multirow[t]{2}{*}{ YMRS score } & $2.1(2.1)$ & $0-7$ \\
\hline & $n$ & $\%$ \\
\hline Gender, female/male & $57 / 23$ & $71 / 29$ \\
\hline Employed & 37 & 46 \\
\hline Bipolar disorder type, I/II & $53 / 27$ & $66 / 34$ \\
\hline Family history of affective disorders & 43 & 54 \\
\hline History of psychosis & 52 & 65 \\
\hline \multicolumn{3}{|l|}{ Current medication class } \\
\hline Lithium & 26 & 33 \\
\hline Anticonvulsants & 47 & 59 \\
\hline Antipsychotics & 59 & 74 \\
\hline Antidepressants & 39 & 49 \\
\hline Anxiolytics & 13 & 16 \\
\hline
\end{tabular}




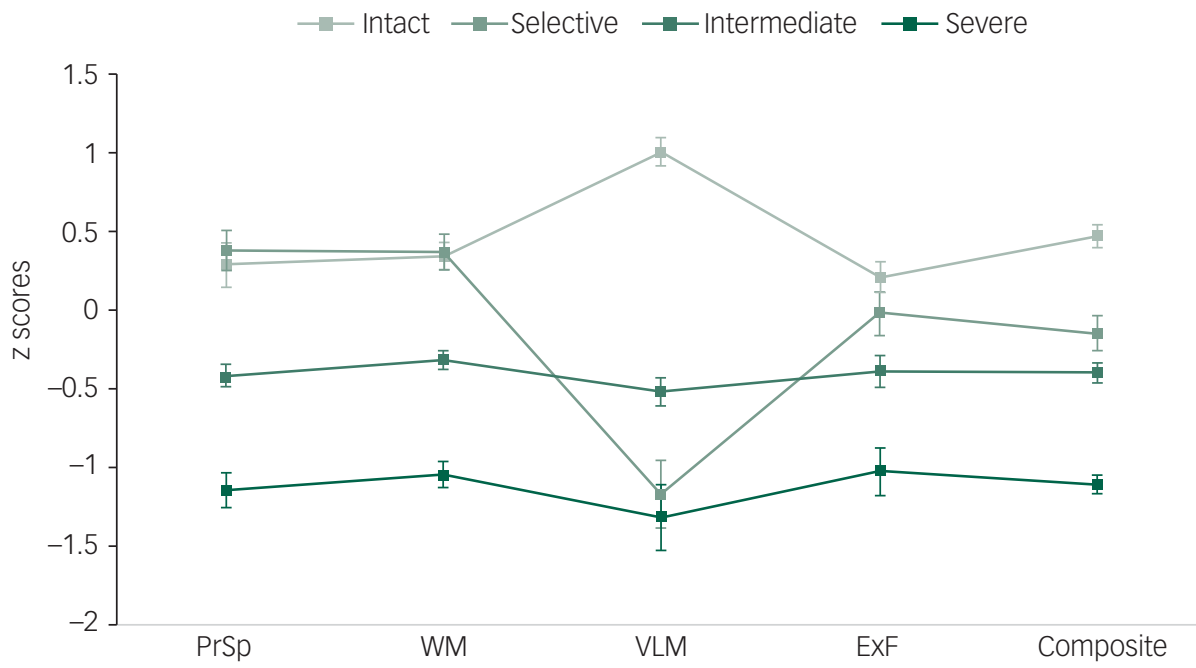

Fig. 1 Cognitive profiles (domain mean and standard error) of the four subgroups (cognitively intact; selective deficit in verbal learning and memory; intermediate impairment across all domains; and severe global impairments). PrSp, processing speed; WM, working memory; VLM, verbal learning and memory; ExF, executive functioning; Composite, composite cognitive score.

normative mean across all domains. The last cluster was characterised by severe impairments exceeding 1 s.d. below the norm in all domains. Figure 1 illustrates the cognitive profiles of the subgroups.

The DFA revealed the presence of two significant discriminant functions, explaining $70 \%$ and $29 \%$ of the cluster membership variance respectively (Wilks' $\lambda=0.11, \chi^{2}=170.5, P<0.001$ and Wilks' $\lambda=0.43, \chi^{2}=63.2 \quad P<0.001$ respectively). Verbal learning and memory were the main contributors to participant classification in function $1(\beta=0.7)$, followed by working memory in function $2(\beta=0.5)$. According to the DFA, $97.5 \%$ of the original grouped participants were correctly classified, suggesting a valid clustering of the sample. A scatterplot of participants per cluster is shown in Fig. 2.

\section{Subgroup cognitive profiles}

Comparisons between subgroups on cognitive measures are presented in Table 2. As anticipated, the MANCOVA revealed a significant main effect of cluster on all clustering domains (all $P<0.001$ ). Post hoc pairwise comparisons corrected for multiple testing (5\% FDR) showed that participants in the severely globally impaired subgroup performed significantly lower in all clustering domains and the composite cognitive score compared with the cognitively intact subgroup $(d=2.4-4.1)$ and compared with the selectively impaired subgroup with the exception of verbal memory $(d=$ 1.7-3.6). Smaller but still largely significant differences were found between participants with intermediate and severe global impairments in all domains but executive functioning $(d=1.3-3.1)$.

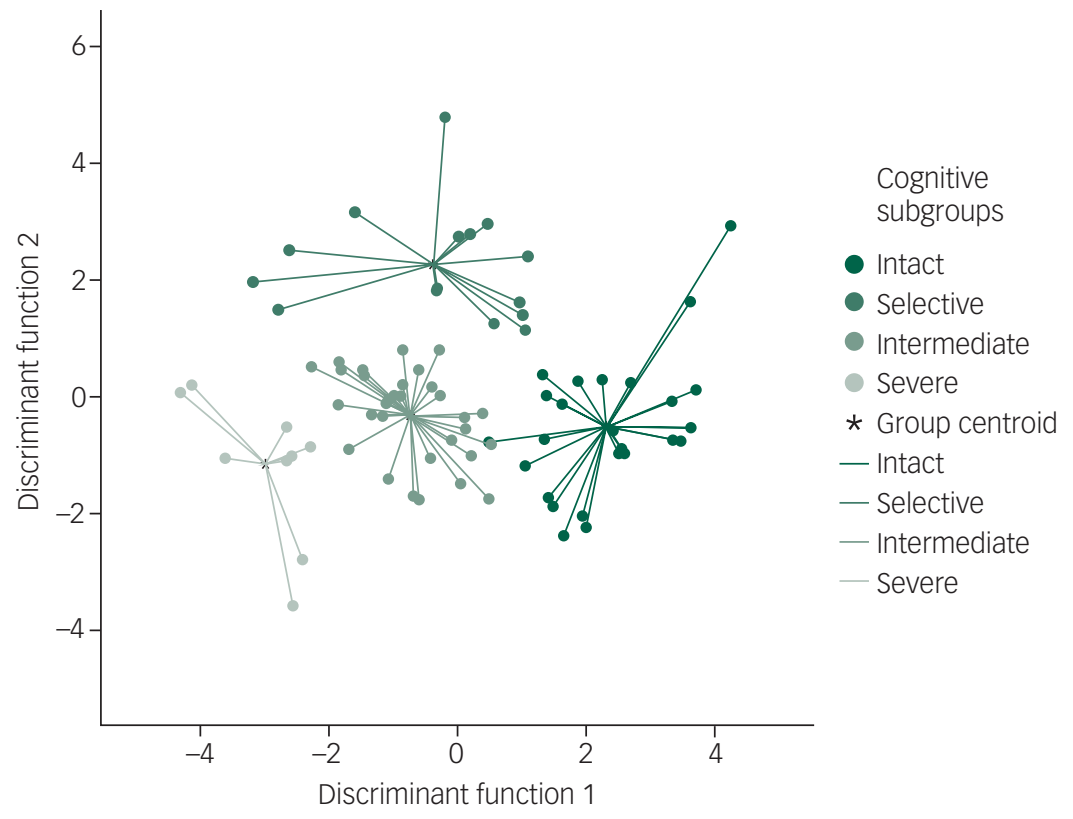

Fig. 2 Graphical agglomeration of the cognitive subgroups (cognitively intact; selective deficit in verbal learning and memory; intermediate impairment across all domains; and severe global impairments). Data plots represent participant scattering and clustering based on the discriminant function values. Group centroids represent mean scores for each cluster. 


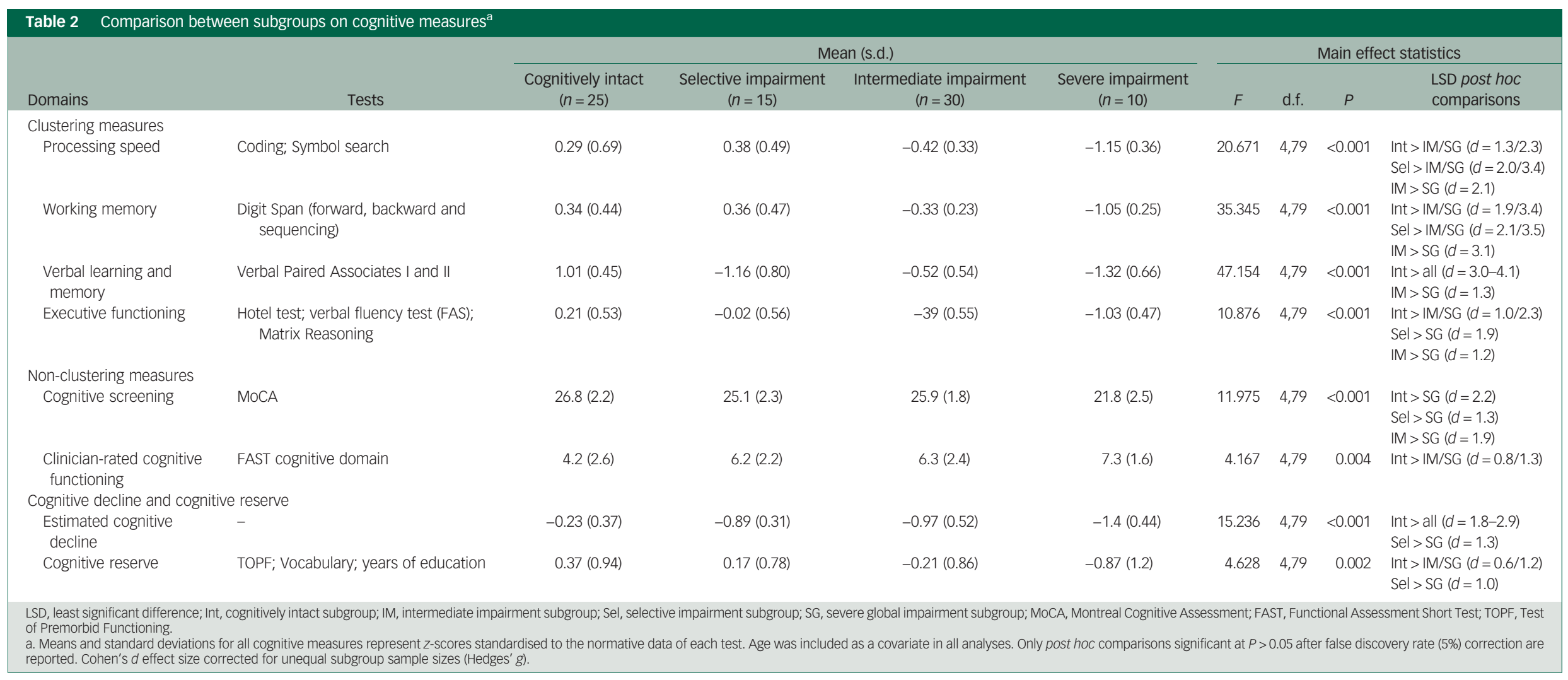




\section{Internally validating cognitive subgroups}

The two non-clustering cognitive measures were significantly correlated with the four clustering domains across the sample. The MoCA showed moderate correlations with working memory $(r=0.29 ; P=0.009)$ and processing speed $(r=0.37 ; P=0.001)$ and moderate to large correlations with executive functioning $(r=0.42 ; P<0.001)$ and verbal memory $(r=0.47 ; P<0.001)$. The cognitive subscale of the FAST was moderately correlated with all domains $(r=0.27-0.37$; all $P<0.01)$ but processing speed. The severe global impairment subgroup performed significantly worse than all other groups on the MoCA $(d=1.3-2.2)$, while both the intermediate impairment $(d=0.8)$ and the severe global impairment $(d=1.3)$ subgroups had poorer FAST scores than the intact subgroup.

\section{Cognitive decline and cognitive reserve}

The MANCOVA for the estimated cognitive decline and the cognitive reserve score revealed significant differences between subgroups for both measures (all $P<0.01$ ). Details are reported in Table 2 . The discrepancy between the current global cognition composite and the estimated premorbid cognitive functioning (TOPF score) indicated a decline for all subgroups, which gradually increased in magnitude from the cognitively intact to the severe impairment subgroup (Fig. 3). Compared with the intact participants, all other subgroups presented with significantly greater cognitive decline $(d=1.8-2.9)$. This difference was also significant between the selective impairment and the severe impairment subgroups $(d=1.3)$.

As hypothesised, the cognitive reserve score was not correlated with age $(r=-0.09 ; P>0.1)$, age at illness onset $(r=-0.15 ; P>0.1)$, illness duration $(r=0.08 ; P>0.1)$ and number of mood episodes $(r=-0.18 ; P>0.1)$. The cognitively intact subgroup had a higher cognitive reserve compared with the intermediate impairment $(d=0.6)$ and the severe impairment $(d=1.2)$ subgroups, while a significant difference was found between the selective impairment and the severe impairment subgroups $(d=1)$. Cognitive reserve showed a small, non-significant correlation with estimated cognitive decline across the sample $(r=-0.2 ; P=0.08)$. However, examining this

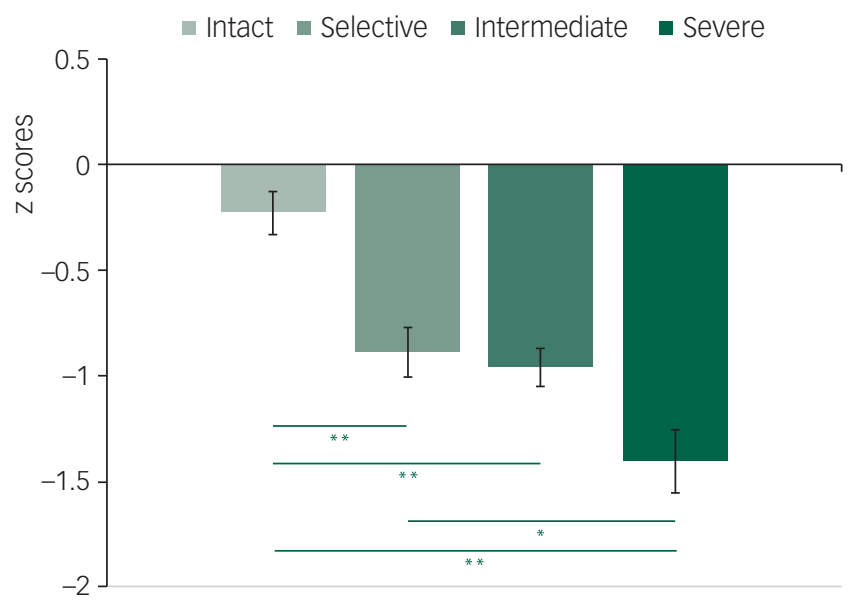

Fig. 3 Estimated postmorbid cognitive decline per subgroup (cognitively intact; selective deficit in verbal learning and memory; intermediate impairment across all domains; and severe global impairments), calculated as the discrepancy between current global cognition composite score and premorbid cognitive functioning (Test of Premorbid Function) score. Significant differences (5\% false discovery rate correction): ${ }^{*} P<0.1,{ }^{* *} P<$ 0.001 . association within each group, cognitive reserve and cognitive decline were strongly correlated for the intact, the intermediate impairment and the severe impairment subgroups $(r=-0.65$ to -0.74 ; all $P<0.05$ ), while the correlation was not significant for the selective impairment subgroup $(r=0.05)$.

\section{Subgroup clinical characteristics}

Table 3 reports the ANOVA results of cluster comparisons for demographic and clinical characteristics. Significant main effects of cluster were found for age, age at diagnosis, the number of psychological therapies previously attended and medication use $(P=0.02-0.04)$. From illness-history variables, only age at onset differed between subgroups, where the selectively and the intermediately impaired participants were diagnosed at an older age compared with the cognitively intact subgroup $(d=1$ and $d=0.8$ respectively). Intact participants had undertaken more psychological therapies than severely impaired participants $(d=1)$ and were taking fewer medications compared with the selectively and intermediately impaired subgroups $(d=0.8)$. A significantly smaller proportion of this subgroup used antipsychotics compared with all other subgroups $(d=0.7)$. No differences among subgroups were detected for any other clinical variables or mood measures.

\section{Discussion}

Using an extensive cognitive battery and hierarchical cluster analysis, this study examined the presence of discrete cognitive profiles in a cohort of euthymic people with bipolar disorder to evaluate a clustering solution independent of mood symptoms. We examined the characteristics of these clusters, specifically related to cognitive reserve. Approximately one-third of participants appeared cognitively intact $(31 \%)$ and another third demonstrated intermediate deficits across all domains (37.5\%), with the remainder presenting a selective impairment in verbal learning and memory (19\%) or showing severe deficits across all domains (12.5\%). The most discriminating cognitive domains for the profiles were verbal learning and memory, followed by working memory and processing speed. Cognitively impaired subgroups presented with greater cognitive decline and poorer cognitive reserve, but few differences were detected in clinical characteristics.

\section{How do the identified cognitive profiles relate to previous findings?}

The four clusters found in this study are in line with previous work on euthymic individuals with bipolar disorder, ${ }^{18,19}$ as well as crossdiagnostic studies including participants from the wider schizophrenia-bipolar disorder spectrum. ${ }^{9}$ The proportion of cognitively intact participants (31\%) is very similar to reports in other studies $(20-45 \%),{ }^{7}$ and above average performance in verbal learning and memory in this subgroup has also been reported. ${ }^{19}$ A relatively small proportion (12.5\%) presented with severe deficits across all domains, previously reported to comprise $10-35 \%$ of people with bipolar disorder. ${ }^{7}$ A possible explanation for the proportion being at the lower end of this range is that our sample included only individuals in full remission, with higher overall premorbid cognitive functioning and more years in education than previous studies, which might indicate a high-performing sample compared with other cohorts (supplementary Table 1, available at https://doi.org/ 10.1192/bjo.2020.111). The other two clusters included participants who either experienced a significant impairment in a single domain but otherwise remained intact (19\%), or participants with intermediate impairment across all domains (37.5\%). These clusters 
correspond to subgroups described as having 'selective' or 'moderate' impairment in previous studies reporting four clusters (15$40 \%)^{7}$

The division of moderately impaired participants into two distinct clusters was the main difference from previous studies reporting three-cluster solutions. One factor affecting potential clustering is the definition of euthymia used for inclusion. In this study we included only individuals in full remission and previous studies with similar definitions also found four-cluster solutions, with subgroups impaired in a single domain or an intermediate impairment across all domains. ${ }^{18,19}$ In contrast, studies with less restrained definitions or including participants in partial remission have mostly reported three-cluster solutions. ${ }^{11,35,36}$ (supplementary Table 1). Although there is no clear explanation for this disparity, it is possible that reduced cognitive performance in otherwise intact domains due to residual depressive symptoms leads to a shared cluster between participants with selective and intermediate deficits.

The performance of each subgroup across the clustering domains suggested largely distinct cognitive profiles, apart from the cognitively intact and the selective impairment subgroups, where the difference was significant only for verbal memory. This distinction was partially validated by the non-clustering measures. Although the clustering and non-clustering cognitive measures showed small to moderate correlations between subgroups, significant subgroup differences validating the identified profiles for this sample were only found between the intact and the severely impaired participants. These two subgroups were clearly separated on both non-clustering measures, whereas differences observed between the selective impairment and intermediate impairment subgroups were minimal.

Despite multiple studies supporting the existence of distinct cognitive profiles among people with bipolar disorder, these results were solely based on behavioural measures analysis, while potential biological underpinnings of cognitive variability remain largely unknown. Recent neuroimaging findings have pointed to weaker interregional connectivity as a neural mechanism possibly underlying cognitive heterogeneity in subgroups of people with bipolar I disorder clustered on the basis of their performance on executive functioning tasks. ${ }^{37}$ Although this study differed from typical cluster-analytic designs in terms of grouping participants according to their response pattern (i.e. encompassing strengths and deficits in their performance) rather than their normative cognitive performance, it did provide initial evidence on the neurological background of distinct cognitive profiles in bipolar disorder.

\section{What is the role of cognitive reserve?}

Reduced premorbid IQ has been associated with the severe impairment subgroup in previous bipolar disorder and cross-diagnostic cluster-analytic studies. ${ }^{10,11}$ Our study cultivates these findings and indicates a linear pattern of increasingly reduced cognitive reserve across subgroups, gradually reducing from the intact to the severe global impairment subgroup. The reverse pattern was observed for the estimated cognitive decline, and the association between cognitive reserve and cognitive decline was significant for all participants but the selectively impaired ones. On the basis of these findings, it is plausible that differential cognitive performance across subgroups is a function of cognitive reserve for the intact, the intermediate impairment and the severe impairment subgroups. This is consistent with the theoretical concept of cognitive reserve in dementia and schizophrenia spectrum or mood disorders, as a protective mechanism against cognitive decline caused by neuropathology itself ${ }^{13,38}$ or against the adverse cognitive effects of certain treatments (e.g. memory performance after electroconvulsive therapy). ${ }^{39}$

Although cognitive decline after symptom onset was evident across all subgroups, the severely impaired subgroup showed the greatest decline and had the poorest cognitive reserve. Cognitive reserve potentially modifies cognitive performance and explains individual differences in the cognitive course of people with bipolar disorder, with worse outcomes for those with poorer reserve. ${ }^{17,40}$ Recent evidence points to a subgroup of people with bipolar disorder sharing genetic and symptomatic characteristics with schizophrenia. ${ }^{41,42}$ This subgroup presents with a cognitive profile comparable to that of people with schizophrenia, including a neurodevelopmental trajectory characterised by poor cognitive reserve. ${ }^{43,44}$ This neurodevelopmental hypothesis is further supported by evidence for the existence of discrete cognitive clusters in young offspring of people with bipolar disorder prior to illness onset. ${ }^{45}$

In our sample, participants with severe deficits across domains potentially represent this subgroup of people with bipolar disorder following a cognitive course defined by reduced cognitive reserve. This argument is strengthened by non-significant or inconsistent findings for subgroup differences in clinical and illness-history characteristics (e.g. diagnostic subtype, illness duration, number of episodes), suggesting that cognitive variability in people with bipolar disorder cannot be entirely attributed to illness progression. Pending confirmation by longitudinal studies, cognitive reserve may be a factor driving disparate illness trajectories and a characteristic that may predict the extent of cognitive impairment in people with bipolar disorder.

\section{Do clusters differ in their clinical characteristics?}

No significant differences were observed between subgroups in demographic characteristics and most illness-history variables, which suggests that subgroup allocation and cognitive variability in our sample was largely independent of clinical characteristics. Likewise, subthreshold depressive and (hypo)manic symptoms were balanced between subgroups, which has been previously reported for cluster-analytic studies in bipolar disorder. ${ }^{7}$

Although the duration of illness and the number of mood episodes did not differ between subgroups, cognitively intact participants were diagnosed at a younger age than participants in the selective impairment and intermediate impairment subgroups. People with bipolar disorder experience significant delays in receiving the correct diagnosis and in receiving treatment. ${ }^{46}$ As a result, differences in age at diagnosis may not reflect differences in age at symptom onset. To examine that further, we computed the duration of untreated illness (DUI), defined as the discrepancy between the time when they first experienced symptoms and when they received their diagnosis, and compared it across subgroups. Although cognitively intact participants received their diagnosis 7 years on average after symptom onset and DUI exceeded 10 years for all other subgroups, this difference was not statistically significant. Thus, our findings do not support that greater DUI is associated with more severe illness course in terms of cognitive impairments. ${ }^{47}$

Subgroup differences were evident in treatment characteristics. To the best of our knowledge, this is the first study reporting that cognitively intact participants had undertaken more psychological therapies than those with severe impairment, but it is unclear whether this reflects a neuroprotective effect of psychological therapies or if it simply reflects the capacity of participants in this subgroup for better treatment access and adherence. A smaller proportion of intact participants were using antipsychotics compared with the other subgroups. This was despite the lack of any significant subgroup differences in history of psychosis and has been previously reported in cluster-analytic studies. ${ }^{18}$ The relationship 
between treatment and potential cognitive outcomes is complex, as greater use of antipsychotics may be an indicator of greater illness severity, particularly regarding increased risk for manic relapse. Studies examining the cognitive course of patients who discontinued or reduced their antipsychotic medication may clarify this question. $^{48}$

\section{Limitations}

This was a cross-sectional study so we cannot speculate on the stability of these cognitive subgroups. Likewise, estimated cognitive decline following illness onset was calculated using cross-sectionally collected measures. In addition, the sample size was relatively small, although it did meet the minimum requirements for a cluster analysis. Therefore, findings on the role of cognitive reserve need to be interpreted with caution and require further replication in larger studies. There is a possibility that effect sizes were inflated owing to our sample size, but we did use a conservative approach for multiple comparison correction to reduce false positives. We therefore consider the identified subgroups and the detected differences reasonable. The neuropsychological battery used was less extensive, with fewer tests per domain compared with some of the previous studies, but cognitive scores did not differ significantly within each domain and the variation in participant scores from the domain mean was small, indicating limited estimation bias. Finally, we did not measure psychiatric comorbidities that could potentially affect cognitive performance.

\section{What are the clinical implications?}

We recently reviewed the literature on cognitive remediation therapies targeting cognitive and functional outcomes for people with bipolar disorder. ${ }^{49}$ Findings are promising but still inconsistent across studies. Cognitive heterogeneity in study samples may underly this inconsistency. Individuals with different cognitive profiles can have different outcomes and some adaptations may be necessary in treatment provision. Different patient clusters may require modifications to adhere to and benefit from treatment, for example longer or more intensive therapy periods for those more severely impaired. ${ }^{50}$ Our findings also suggest that considering the concept of cognitive reserve might be relevant in the context of designing and delivering cognitive interventions, either as a potential treatment target or as a factor enabling treatment engagement and outcomes. Clinical services for people with bipolar disorder should introduce cognitive assessment into their screening process for new or recurring patients. The cognitive domain mostly contributing to differentiation of the four cognitive profiles was verbal learning and memory, and previous research has associated verbal memory performance with future functional outcomes. ${ }^{51}$ Hence, it might be a suitable domain for clinical services to screen in order to quickly and effectively differentiate between cognitively intact and compromised patients.

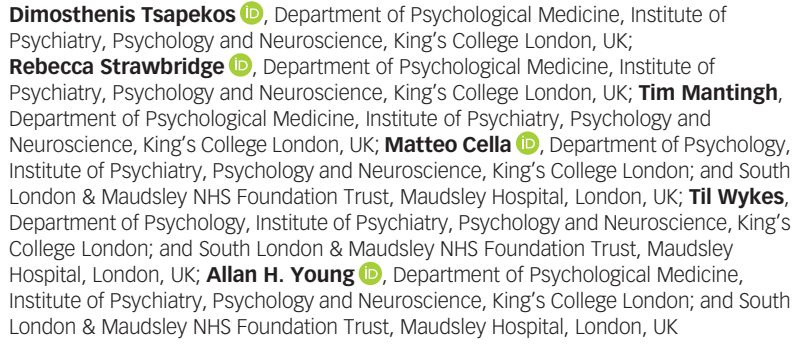

Correspondence: Dimosthenis Tsapekos. Email: dimosthenis.tsapekos@kcl.ac.uk

First received 10 Jun 2020, final revision 28 Aug 2020, accepted 17 Sep 2020

\section{Supplementary material}

Supplementary material is available online at https://doi.org/10.1192/bjo.2020.111.

\section{Data availability}

The anonymised data-set is available from the corresponding author on request.

\section{Acknowledgements}

We are grateful to all CRiB study participants and team members.

\section{Author contributions}

D.T. and A.H.Y. conceived and planned this study. D.T., R.S. and T.M. were involved in data collection. D.T. carried out the analysis and prepared the first manuscript, with support from R.S. and T.M.. M.C., T.W. and A.H.Y. supervised the project. All authors provided feedback and contributed to the final manuscript.

\section{Funding}

This paper represents independent research part-funded by the National Institute for Health Research (NIHR) Maudsley Biomedical Research Centre at South London and Maudsley NHS Foundation Trust and King's College London. The views expressed are those of the authors and not necessarily those of the NIHR or the Department of Health and Social Care. D.T. would like to acknowledge that this paper was supported by the Onassis Foundation (scholarship: F ZO077-1/2018-2019). A.H.Y. and T.W. would like to acknowledge their NIHR Senior Investigator awards.

\section{Declaration of interest}

A.H.Y. reports paid lectures and advisory board membership for the following companies with drugs used in affective and related disorders: AstraZeneca, Eli Lilly, Lundbeck, Sunovion, Servier, LivaNova, Allegan, Bionomics, Sumitomo, Dainippon Pharma and Janssen; consultancy to Johnson \& Johnson and LivaNova; principal investigator on the Restore-Life VNS registry study funded by LivaNova and on ESKETINTRD3004 funded by Janssen. R.S. reports a paid lecture from Lundbeck.

ICMJE forms are in the supplementary material, available online at http://dx.doi.org/10. 1192/bjo.2020.111.

\section{References}

1 Arts B, Jabben N, Krabbendam L, van Os J. Meta-analyses of cognitive functioning in euthymic bipolar patients and their first-degree relatives. Psychol Med 2008; 38: 771-85

2 Torres IJ, Boudreau VG, Yatham LN. Neuropsychological functioning in euthymic bipolar disorder: a meta-analysis. Acta Psychiatr Scand 2007; 116: 17-26.

3 Bourne C, Aydemir O, Balanza-Martinez V, Bora E, Brissos S, Cavanagh JT, et al. Neuropsychological testing of cognitive impairment in euthymic bipolar disorder: an individual patient data meta-analysis. Acta Psychiatr Scand 2013; 128: 149-62.

4 Léda-Rêgo G, Bezerra-Filho S, Miranda-Scippa Â. Functioning in euthymic patients with bipolar disorder: a systematic review and meta-analysis using the Functioning Assessment Short Test. Bipolar Disord 2020; 22: 569-81.

5 Reichenberg A, Harvey PD, Bowie CR, Mojtabai R, Rabinowitz J, Heaton RK, et al. Neuropsychological function and dysfunction in schizophrenia and psychotic affective disorders. Schizophr Bull 2009; 35: 1022-9.

6 Burdick KE, Goldberg TE, Cornblatt BA, Keefe RS, Gopin CB, Derosse P, et al. The MATRICS consensus cognitive battery in patients with bipolar I disorder. Neuropsychopharmacology 2011; 36: 1587-92.

7 Green MJ, Girshkin L, Kremerskothen K, Watkeys O, Quidé Y. A systematic review of studies reporting data-driven cognitive subtypes across the psychosis spectrum. Neuropsychol Rev [Epub ahead of print] 18 Dec 2019. Available from: https://doi.org/10.1007/s11065-019-09422-7.

8 Van Rheenen TE, Lewandowski KE, Tan EJ, Ospina LH, Ongur D, Neill E, et al. Characterizing cognitive heterogeneity on the schizophrenia-bipolar disorder spectrum. Psychol Med 2017; 47: 1848-64.

9 Lewandowski KE, Sperry SH, Cohen BM, Ongur D. Cognitive variability in psychotic disorders: a cross-diagnostic cluster analysis. Psychol Med 2014; 44: 3239-48

10 Karantonis JA, Rossell SL, Carruthers SP, Sumner P, Hughes M, Green MJ, et al. Cognitive validation of cross-diagnostic cognitive subgroups on the schizophrenia-bipolar spectrum. J Affect Disord 2020; 266: 710-21.

11 Burdick KE, Russo M, Frangou S, Mahon K, Braga RJ, Shanahan M, et al. Empirical evidence for discrete neurocognitive subgroups in bipolar disorder: clinical implications. Psychol Med 2014; 44: 3083-96.

12 Stern $\mathrm{Y}$. What is cognitive reserve? Theory and research application of the reserve concept. J Int Neuropsychol Soc 2002; 8: 448-60. 
13 Stern Y. Cognitive reserve in ageing and Alzheimer's disease. Lancet Neurol 2012; 11: 1006-12.

14 Sumowski JF, Chiaravalloti N, DeLuca J. Cognitive reserve protects against cognitive dysfunction in multiple sclerosis. J Clin Exp Neuropsychol 2009; 31: 913-26.

15 Stern $\mathrm{Y}$, Habeck $\mathrm{C}$, Moeller J, Scarmeas $\mathrm{N}$, Anderson $\mathrm{KE}$, Hilton $\mathrm{HJ}$, et al. Brain networks associated with cognitive reserve in healthy young and old adults. Cereb Cortex 2004; 15: 394-402.

16 Van Rheenen TE, Cropley V, Fagerlund B, Wannan C, Bruggemann J, Lenroot RK et al. Cognitive reserve attenuates age-related cognitive decline in the context of putatively accelerated brain ageing in schizophrenia-spectrum disorders. Psychol Med 2020: 50: 1475-89.

17 Grande I, Sanchez-Moreno J, Sole B, Jimenez E, Torrent C, Bonnin CM, et al. High cognitive reserve in bipolar disorders as a moderator of neurocognitive impairment. J Affect Disord 2017; 208: 621-7.

18 Bora E, Hidiroglu C, Ozerdem A, Kacar OF, Sarisoy G, Civil Arslan F, et al Executive dysfunction and cognitive subgroups in a large sample of euthymic patients with bipolar disorder. Eur Neuropsychopharmacol 2016; 26: 1338-47.

19 Roux P, Raust A, Cannavo AS, Aubin V, Aouizerate B, Azorin JM, et al. Cognitive profiles in euthymic patients with bipolar disorders: results from the FACE-BD cohort. Bipolar Disord 2017; 19: 146-53.

20 Strawbridge R, Fish J, Halari R, Hodsoll J, Reeder C, Macritchie K, et al. The Cognitive Remediation in Bipolar (CRiB) pilot study: study protocol for a randomised controlled trial. Trials 2016; 17: 371.

21 Strawbridge R, Tsapekos D, Hodsoll J, Mantingh T, Yalin N, Mccrone P, et al Cognitive remediation therapy for patients with bipolar disorder: a randomised proof-of-concept trial. Bipolar Disord [Epub ahead of print] 24 Jun 2020. Available from: https://doi.org/10.1111/bdi.12968

22 Sheehan DV, Lecrubier $Y$, Sheehan $\mathrm{KH}$, Amorim $\mathrm{P}$, Janavs J, Weiller $\mathrm{E}$, et al. The Mini-International Neuropsychiatric Interview (M.I.N.I.): the development and validation of a structured diagnostic psychiatric interview for DSM-IV and ICD-10. J Clin Psychiatry 1998; 59(suppl 20): 22-33.

23 Hamilton M. A rating scale for depression. J Neurol Neurosurg Psychiatry 1960; 23: $56-62$.

24 Young RC, Biggs JT, Ziegler VE, Meyer DA. A rating scale for mania: reliability, validity and sensitivity. Br J Psychiatry 1978; 133: 429-35.

25 Manly T, Hawkins K, Evans J, Woldt K, Robertson IH. Rehabilitation of executive function: facilitation of effective goal management on complex tasks using periodic auditory alerts. Neuropsychologia 2002; 40: 271-81.

26 Wechsler D. Wechsler Memory Scale: WMS-IV; Technical and Interpretive Manual. Pearson, 2009.

27 Dumont R, Willis JO, Veizel K, Zibulsky J. Wechsler Abbreviated Scale of Intelligence. In Encyclopedia of Special Education (4th edn). John Wiley \& Sons, 2014 (https://doi.org/10.1002/9781118660584.ese2519).

28 Wechsler D. Wechsler Adult Intelligence Scale-Fourth Edition (WAIS-IV). Psychological Corporation, 2014.

29 Delis D. Delis-Kaplan Executive Function Scale (D-KEFS). Psychological Corporation, 2001

30 Nasreddine ZS, Phillips NA, Bédirian V, Charbonneau S, Whitehead V, Collin I, et al. The Montreal Cognitive Assessment, MoCA: a brief screening tool for mild cognitive impairment. J Am Geriatr SOC 2005; 53: 695-9.

31 Rosa AR, Sánchez-Moreno J, Martínez-Aran A, Salamero M, Torrent C, Reinares M, et al. Validity and reliability of the Functioning Assessment Short Test (FAST) in bipolar disorder. Clin Pract Epidemol Ment Health 2007; 3(1): 5

32 Wechsler D. Test of Premorbid Functioning - UK Version (TOPF UK). Pearson, 2011.

33 Ryan JJ, Sattler JM, Lopez SJ. Age effects on Wechsler Adult Intelligence Scale-III subtests. Arch Clin Neuropsychol 2000; 15: 311-7.

34 Dolnicar S. A review of unquestioned standards in using cluster analysis for data-driven market segmentation. In $C D$ Conference Proceedings of the
Australian and New Zealand Marketing Academy Conference 2002 (ANZMAC 2002), Deakin University, Melbourne, 2-4 December. Deakin University, 2002.

35 Jensen JH, Knorr U, Vinberg M, Kessing LV, Miskowiak KW. Discrete neurocognitive subgroups in fully or partially remitted bipolar disorder: associations with functional abilities. J Affect Disord 2016; 205: 378-86.

36 Russo $M$, Van Rheenen $T E$, Shanahan $M$, Mahon $K$, Perez-Rodriguez MM, Cuesta-Diaz A, et al. Neurocognitive subtypes in patients with bipolar disorder and their unaffected siblings. Psychol Med 2017; 47: 2892-905.

37 Kollmann B, Yuen K, Scholz V, Wessa M. Cognitive variability in bipolar I disorder: a cluster-analytic approach informed by resting-state data. Neuropharmacology 2019: 156: 107585.

38 Watson A, Joyce E. Cognitive reserve and neuropsychiatric disorders. Curr Opin Behav Sci 2015; 4: 142-6.

39 Legendre SA, Stern RA, Solomon DA, Furman MJ, Smith KE. The influence of cognitive reserve on memory following electroconvulsive therapy. J Neuropsychiatry Clin Neurosci 2003; 15: 333-9.

40 Anaya C, Torrent C, Caballero FF, Vieta E, del Mar Bonnin C, Ayuso-Mateos JL, et al. Cognitive reserve in bipolar disorder: relation to cognition, psychosocial functioning and quality of life. Acta Psychiatr Scand 2016; 133: 386-98.

41 Bora E, Pantelis C. Meta-analysis of cognitive impairment in first-episode bipolar disorder: comparison with first-episode schizophrenia and healthy controls. Schizophr Bull 2015; 41: 1095-104.

42 Service SK, Vargas Upegui C, Castaño Ramírez M, Port AM, Moore TM, Munoz Umanes $\mathrm{M}$, et al. Distinct and shared contributions of diagnosis and symptom domains to cognitive performance in severe mental illness in the Paisa population: a case-control study. Lancet Psychiatry 2020; 7: 411-9.

43 Bora E. Developmental trajectory of cognitive impairment in bipolar disorder: comparison with schizophrenia. Eur Neuropsychopharmacol 2015; 25: $158-68$.

44 Van Rheenen TE, Lewandowski KE, Bauer IE, Kapczinski F, Miskowiak K, Burdick $\mathrm{KE}$, et al. Current understandings of the trajectory and emerging correlates of cognitive impairment in bipolar disorder: an overview of evidence. Bipolar Disord 2020; 22: 13-27.

45 Bora E, Can G, Ildız A, Ulas G, Ongun $\mathrm{CH}$, Inal NE, et al. Neurocognitive heterogeneity in young offspring of patients with bipolar disorder: the effect of putative clinical stages. J Affect Disord 2019; 257: 130-5.

46 Dagani J, Signorini G, Nielssen O, Bani M, Pastore A, Girolamo GD, et al. Metaanalysis of the interval between the onset and management of bipolar disorder. Can J Psychiatry 2016; 62: 247-58.

47 Drancourt N, Etain B, Lajnef M, Henry C, Raust A, Cochet B, et al. Duration of untreated bipolar disorder: missed opportunities on the long road to optimal treatment. Acta Psychiatr Scand 2013; 127: 136-44.

48 Albert N, Randers L, Allott K, Jensen HD, Melau M, Hjorthøj C, et al. Cognitive functioning following discontinuation of antipsychotic medication. A naturalistic sub-group analysis from the OPUS II trial. Psychol Med 2018; 49 : $1138-47$

49 Tsapekos D, Seccomandi B, Mantingh T, Cella M, Wykes T, Young AH. Cognitive enhancement interventions for people with bipolar disorder: a systematic review of methodological quality, treatment approaches, and outcomes. Bipolar Disord 2020; 22: 216-30.

50 Tsapekos D, Strawbridge R, Cella M, Wykes T, Young AH. Do we really need a "new" cognitive therapy for bipolar disorder? Paradigm refinements and treatment mechanisms for cognitive remediation. Bipolar Disord 2020; 22: 213-5.

51 de Mar Bonnin C, Gonzalez-Pinto A, Sole B, Reinares M, Gonzalez-Ortega I, Alberich $S$, et al. Verbal memory as a mediator in the relationship between subthreshold depressive symptoms and functional outcome in bipolar disorder. J Affect Disord 2014; 160: 50-4.

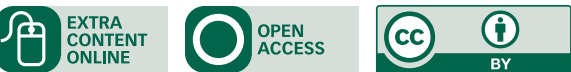

